\title{
Tumor-associated macrophages: from basic research to clinical application
}

\author{
Li Yang ${ }^{1,2}$ and Yi Zhang ${ }^{1,2,3^{*}}$
}

\begin{abstract}
The fact that various immune cells, including macrophages, can be found in tumor tissues has long been known. With the introduction of concept that macrophages differentiate into a classically or alternatively activated phenotype, the role of tumor-associated macrophages (TAMs) is now beginning to be elucidated. TAMs act as "protumoral macrophages," contributing to disease progression. TAMs can promote initiation and metastasis of tumor cells, inhibit antitumor immune responses mediated by $T$ cells, and stimulate tumor angiogenesis and subsequently tumor progression. As the relationship between TAMs and malignant tumors becomes clearer, TAMs are beginning to be seen as potential biomarkers for diagnosis and prognosis of cancers, as well as therapeutic targets in these cases. In this review, we will discuss the origin, polarization, and role of TAMs in human malignant tumors, as well as how TAMs can be used as diagnostic and prognostic biomarkers and therapeutic targets of cancer in clinics.
\end{abstract}

Keywords: Tumor-associated macrophages (TAMs), Tumor microenvironment, Protumoral activities, Biomarker, Therapeutic target

\section{Background}

Non-resolving inflammation in a tumor microenvironment is a hallmark of cancer [1, 2]. Leukocytes, fibroblasts, and vascular endothelial cells together form a tumor microenvironment, with immune cells representing its major component. These immune cells interact with tumor cells to influence the initiation, growth, and metastasis of tumors [3]. Tumor-associated macrophages (TAMs), specifically, are often prominent immune cells that orchestrate various factors in the tumor microenvironment $[4,5]$.

In general, monocytes/macrophages can be polarized to M1 or M2 macrophages. Classically activated macrophages, also known as M1-polarized macrophages, are activated by cytokines such as interferon- $\gamma$, produce proinflammatory and immunostimulatory cytokines (e.g., interleukin [IL]-12 and IL-23), and are involved in helper $\mathrm{T}$ cell $(\mathrm{Th}) 1$ responses to infection. TAMs are thought to more closely resemble M2-polarized macrophages [6],

\footnotetext{
*Correspondence: yizhang@zzu.edu.cn

'Biotherapy Center, The First Affiliated Hospital of Zhengzhou University,

No.1 Jianshe East Road, Zhengzhou 450052, Henan Province, China

${ }^{2}$ Cancer Center, The First Affiliated Hospital of Zhengzhou University, No.1

Jianshe East Road, Zhengzhou 450052, Henan Province, China

Full list of author information is available at the end of the article
}

also known as alternatively activated macrophages, which are activated by Th2 cytokines (e.g., interleukin (IL)-4, IL-10, and IL-13). TAMs play an important role in connecting inflammation with cancer. TAMs can promote proliferation, invasion, and metastasis of tumor cells, stimulate tumor angiogenesis, and inhibit antitumor immune response mediated by $\mathrm{T}$ cells, followed by the promotion of tumor progression [6].

With the unraveling of the relationship between TAMs and malignant tumors, TAMs are now being recognized as potential biomarkers for diagnosis and prognosis of cancer, as well as potential therapeutic targets for cancer. In this review, we summarize how TAMs are involved in tumor progression and discuss the clinical significance of TAMs in diagnosis and prognosis of cancers and their use as therapeutic targets in these cases.

\section{Origins of TAMs}

The original understanding of tissue macrophages was that they were solely derived from bone marrow. However, lung alveolar and peritoneal macrophages, Kupffer cells, epidermal Langerhans cells, and brain microglia derived from primitive yolk sac precursors are referred to as tissue-resident macrophages, and they are 
locally self-maintained. The contribution of locally proliferating macrophages to the pool of TAMs was demonstrated in a Her2/Neu-driven mammary carcinoma animal study [7]. Although there is evidence that all kinds of macrophages can coexist in tumors, recruited macrophages may account for the majority of TAMs and the respective contributions of these macrophages to the various stages of progression in many different tumors cannot be currently quantified. Further studies to characterize TAMs in different human cancers are needed (Fig. 1).

Peripheral blood monocytes from bone marrow are recruited locally and differentiate into TAMs in response to chemokines and growth factors produced by stromal and tumor cells in the tumor microenvironment.
Colony-stimulating factor (CSF) 1 is the master regulator and chemotactic factor for most populations of macrophages, whether they are derived from the yolk sac or bone marrow [8]. In a polyoma middle $\mathrm{T}$ oncoprotein model, the binding of chemokine ( $\mathrm{C}-\mathrm{C}$ motif) ligand (CCL) 2 to chemokine (C-C motif) receptor (CCR) 2 directly mediated monocyte recruitment to the primary tumor and metastases [9]. In a xenograft model, vascular endothelial growth factor A (VEGFA) recruited monocytes that differentiated into TAMs in the presence of IL-4 and the absence of these TAMs inhibited tumor growth, invasion, proliferation, and angiogenesis [10]. In human breast cancer models, binding of CCL18 to its receptor PITPNM3 mediated the recruitment of macrophages in collaboration with CSF2 [11]. In colon cancer

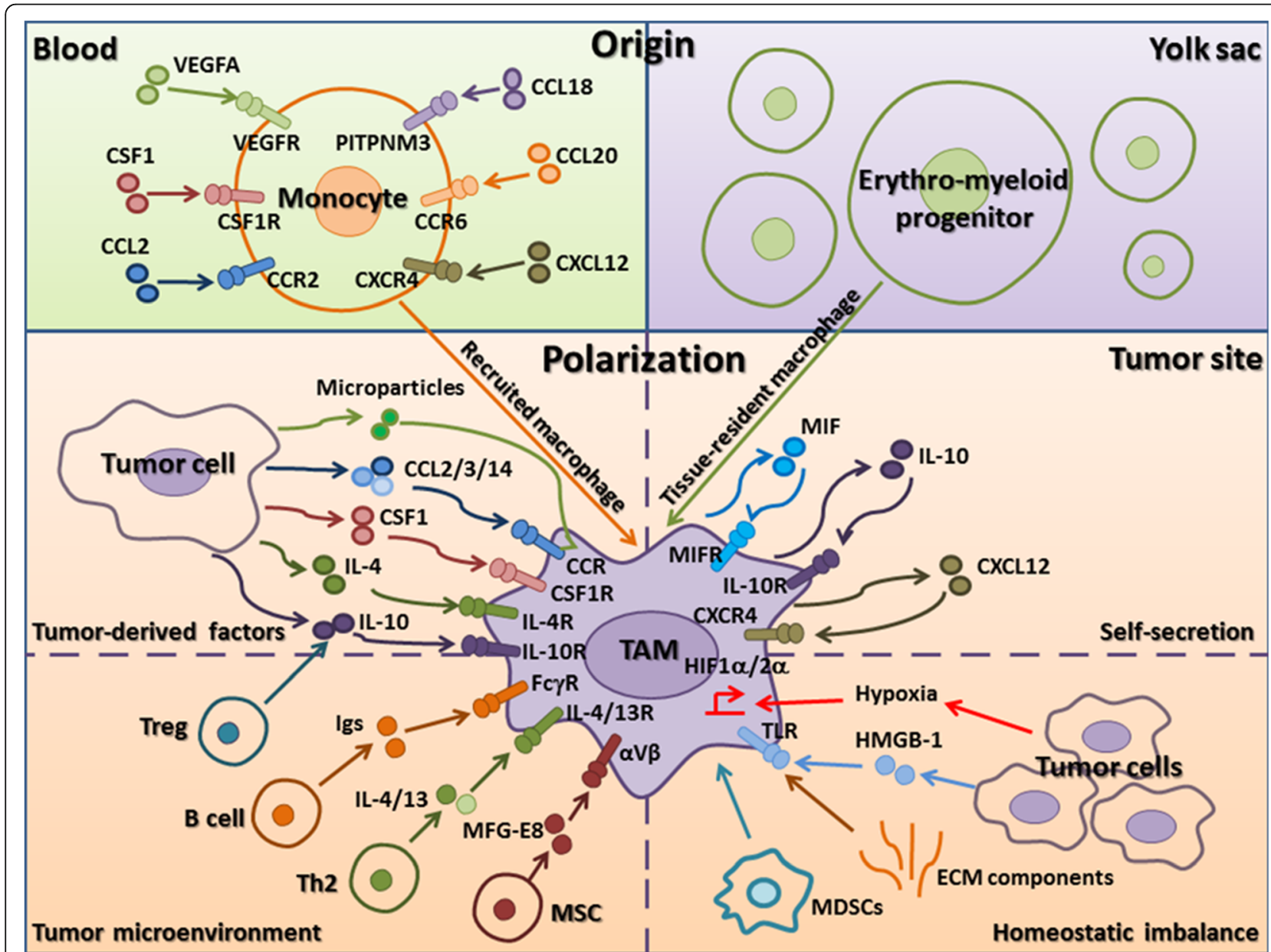

Fig. 1 The origin and polarization of TAMs in tumor microenvironments. Recruited macrophages from blood (green) and tissue-resident macrophages from the yolk sac (purple) coexist in tumors. Recruited macrophages represent the majority of TAMs. Peripheral blood monocytes are recruited locally and differentiate into macrophages in response to various chemokines and growth factors produced by stromal and tumor cells in the tumor microenvironment (CCL2, CSF1, VEGFA, CCL18, CCL20, and CXCL12). Factors that promote the polarization of TAMs to a protumor phenotype can be subdivided into those actively produced by tumor cells (microparticles, CCL2/3/4, CSF1, IL-4, IL-10), those derived from immune system components (Treg-derived IL-10, B cell-derived Igs, Th2-derived IL-4/13, and MSC-derived MFG-E8), those secreted by TAMs (MIF, IL-10, CXCL12), and those resulting from tissue stress (hypoxia, tumor-derived HMGB-1, ECM components) (orange). In addition, TAMs can also be differentiated from myeloid-derived suppressor cells in the leukemic stem cell niche 
models, macrophage recruitment was mediated by CCL20 binding to its receptor CCR6 [12], the ablation of the chemokine resulted in the loss of monocytes and/or TAMs and inhibition of the malignancy. The accumulation of TAMs in response to CXC chemokine receptor type (CXCR) 4/CXC motif chemokine ligand (CXCL) 12 has been shown to contribute to B16 melanoma progression [13] (Fig. 1).

\section{Polarization of TAMs}

Based on their functions within the tumor microenvironment, TAMs are generally characterized as M2-like macrophages, which express higher levels of anti-inflammatory cytokines, scavenging receptors, angiogenic factors, and proteases than that in M1-type macrophages. These antiinflammatory cytokines can reprogram the immunosuppressive microenvironment and then promote tumor progression with TAM-derived angiogenic factors, and proteases by multiple ways described in "TAMs promote cancer progression." TAMs do not become polarized by virtue of their location per se but instead receive signals from the particular microenvironment in which they reside. Currently, a variety of long non-coding RNAs has been demonstrated to impair the function and development of monocyte-macrophages [14]. Moreover, the factors affecting the polarization of TAMs are discussed in detail below (Fig. 1).

\section{Tumor-derived factors}

Several factors produced by tumor cells can reduce macrophage polarization (Fig. 1). Colon cancer cellderived CSF1 has been shown to drive the recruitment and reeducation of macrophages [15]. Chemokines CCL2, 3, and 14 stimulate macrophage proliferation and polarization in multiple myelomas [16]. IL-10 inhibits the production of pro-inflammatory cytokines and chemokines in macrophages [17]. IL-4 also works in synergy with CSF1 to induce M2-polarized macrophages [18]. Recent evidence indicates that tumor cell-derived microparticles mediate the polarization of TAMs for tumor progression [19]. In addition, prostate cancer-derived cathelicidin-related antimicrobial peptide reeducates macrophages to M2-like phenotype [20]. Hypoxic cancer cell-derived Oncostatin $M$ and Eotaxin differentiate macrophages into M2-polarized phenotype [21]. Soluble MHC I chain-related molecule skews macrophages to immune suppressive alternative phenotype through activation of STAT3 [22].

\section{Tumor microenvironment}

Once monocytes in peripheral blood are recruited to the tumor, the tumor environment rapidly promotes their differentiation into TAMs (Fig. 1). Consistent with the original description of alternative activation, the type 2 cytokine IL-4 secreted from Th2-polarized CD4 ${ }^{+}$cells [23], IL-10 derived from regulatory $\mathrm{T}$ cells (Tregs) [24], and immunoglobulin (Ig) from B cells [25] regulate macrophage polarization to the protumor phenotype. IL13 from Th2 cells may have similar effects on TAM polarization because of overlapping IL-13 and IL-4 signaling cascades that lead to signal transduction and transcription (STAT) 6 activation, although this is yet to be proven in vivo [26]. In addition, mesenchymal stromal cell-derived milk fat globule-epithelial growth factor 8 protein (MFG-E8) [27] has been shown to enhance M2 polarization of macrophages.

\section{Self-secretion}

Recently, migration inhibitory factor (MIF) from macrophages was reported to be an important determinant of TAM polarization in melanoma-bearing mice [28]. MIF deficiency or treatment with an MIF antagonist was shown to attenuate tumor-induced TAM polarization and reduce the expression of proangiogenic genes in TAMs. In addition, tumor-infiltrated macrophages could produce IL-10 to promote TAMs self-polarization [29]. Another study found that autocrine CXCL12 production modulated differentiation of monocytes toward a distinct program with proangiogenic and immunosuppressive functions [30] (Fig. 1).

\section{Homeostatic imbalance}

Hypoxia seems to promote malignant conversion and metastasis, which is mediated primarily through hypoxiainducible factor (HIF)- $1 \alpha$ and HIF-2 $\alpha$. Both of these factors can also regulate macrophage function [31]. The presence of high-mobility group box 1 protein (HMGB1), extracellular ATP, and other normally intracellular molecules is detected by a class of receptors on the surface of macrophages called Toll-like receptors (TLRs). Both TLR2 and TLR6 signaling can promote lung cancer progression by inducing tumor necrosis factor- $\alpha$ (TNF- $\alpha$ ) production of macrophages [32]. Tumor-derived extracellular matrix (ECM) components, including biglycan and hyaluronan, are potentially important factors in directing TAM polarization via TLR2 and TLR4 [33]. Crucially, these ECM components do not bind to TLRs in non-inflamed tissue but become TLR ligands following protease cleavage or interaction with reactive oxygen or nitrogen species, thereby forming putative sensory pathways for the detection of inflammation and tissue disruption. In addition, TAMs can also be differentiated from myeloidderived suppressor cells (MDSCs) in the leukemic stem cell niche [34] (Fig. 1).

\section{TAMs promote cancer progression}

TAMs play particular functional roles in tumor progression, including cancer initiation and promotion, immune 
suppression, metastasis, establishing a premalignant niche, and angiogenesis. Each of these functions is described below (Fig. 2).

\section{Cancer initiation and promotion}

TAMs connect inflammation and cancer. In 2009, cancerrelated inflammation was first defined as a hallmark of cancer. Activated macrophages work in concert with other immune cells in this type of immune response. Evidences suggest that an inflammatory microenvironment promotes genetic instability within developing tumor epithelial cells and infiltrating or resident immune cells such as macrophages in inflammatory microenvironments. Recently, the presence of TAM-derived inflammatory cytokines IL-23 and IL-17 has been shown to be closely associated with cancer progression [35]. Kupffer cells can provide essential mitogens for the promotion of hepatocellular carcinoma through a nuclear factor $\mathrm{\kappa B}(\mathrm{NF}-\mathrm{kB})$-dependent signaling mechanism, because its ablation reduced tumor burden [36]. Recent data indicates that TAM-derived IL-6 promotes the occurrence and development of hepatocellular carcinoma via STAT3 signaling [37]. These results suggest that tumor-infiltrated macrophages play an important role in cancer initiation and promotion (Fig. 2).

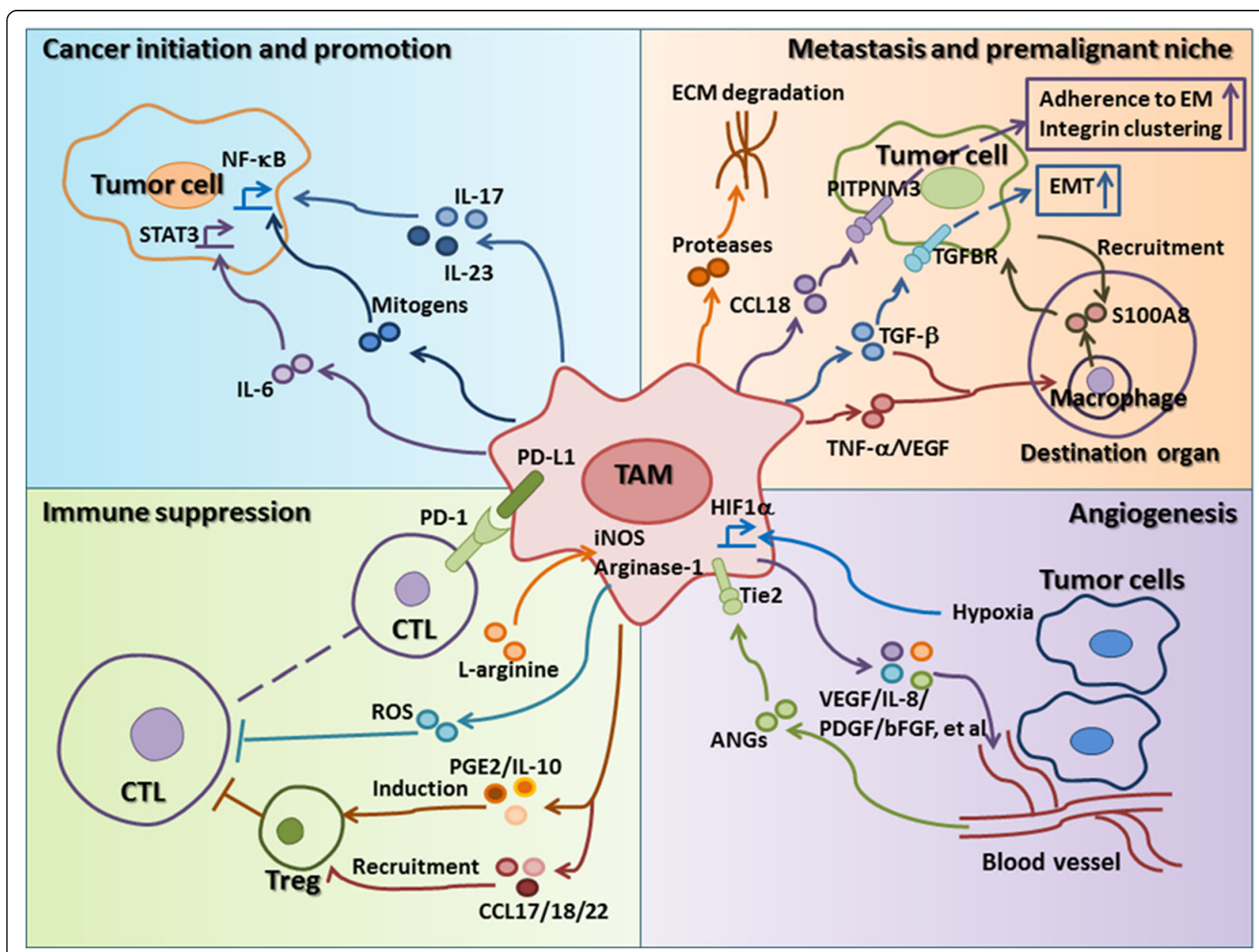

Fig. 2 The effects of TAMs on tumor progression. The protumor functions of TAMs include cancer initiation and promotion (blue), immune suppression (green), metastasis, establishment of a premalignant niche (orange), and promotion of angiogenesis (purple). (1) TAMs can produce cytokines such as IL-6/IL-17/IL-23 or mitogens to induce the initiation and progression of cancer via the NF-KB or STAT3 signaling pathway in tumor cells. (2) Suppression of CTL proliferation by TAMs is at least partly dependent on metabolism of L-arginine via iNOS or arginase I, which results in ROS production. TAMs inhibit CTL responses via PD1/PD-L1 signaling pathway. TAM-derived PGE2 and IL-10 promote the induction of Tregs, and TAM-derived CCL17/18/22 recruit Tregs, which results in CTL suppression. (3) Neoplastic cell invasion of ectopic tissue can be promoted through protease-dependent ECM remodeling that may directly affect neoplastic migration or the premalignant niche. TAM-derived CCL18 promotes tumor metastasis by triggering integrin clustering and enhancing their adherence to extracellular matrix (EM) in tumor cells. TAM-derived TGF- $\beta$ plays important roles in initiation and progression of the EMT. TAMs-derived TNF- $\alpha$, VEGF, and TGF- $\beta$ can transport through the bloodstream to destination organs, where they induce macrophages to produce S100A8, which further recruits tumor cells to these organs and promotes the formation of metastatic foci. (4) Hypoxia induces HIF-1a expression in TAMs and further regulates the transcription of many genes associated with angiogenesis. Subsets of Tie2 ${ }^{+}$TAMs can interact with mural cells/pericytes to regulate vascular structure 


\section{Immune suppression}

TAMs are the major immunoregulatory cells in tumors, and they participate in inhibiting cytotoxic $\mathrm{T}$ lymphocyte (CTL) responses in tumor microenvironments (Fig. 2). In murine tumor models, suppression of $\mathrm{CD}^{+} \mathrm{T}$ cell proliferation by TAMs is at least partly dependent on metabolism of L-arginine via inducible nitric oxide synthase (iNOS) or arginase I, which results in the production of reactive oxygen species (ROS) [38]. IL-10 produced by TAMs can induce the expression of costimulatory molecule PD-L1 in monocytes, which can inhibit CTL responses [39]. In addition, TAM-derived prostaglandin E2 (PGE2), IL-10, and indoleamine 2,3-dioxygenase play important roles in the induction of Tregs and TAM-derived CCL17, CCL18, and CCL22 are chemotactic factors for Tregs [40], which results in the suppression of $\mathrm{T}$ cells in the tumor microenvironment.

\section{Metastasis and premalignant niche}

The most comprehensively described mechanism by which TAMs promote solid tumor development is to provide factors that enhance metastasis and the establishment of a premalignant niche of malignant cells (Fig. 2).

In human xenograft models, CCL18 is also required for tumor cell invasion and metastasis, playing a role in integrin clustering [41]. Migration on and through the ECM is necessary for tumor cells metastasis, and TAMs are believed to promote tumor cell migration/invasion through the ECM [42]. TAMs can produce proteases, including cathepsin B, matrix metallopeptidase (MMP) 2, MMP7, and MMP9, and cleave the ECM, thereby providing conduits for tumor cells.

The epithelial-mesenchymal transition (EMT) is an important result of the interaction between TAMs and tumor cells. EMT plays a fundamental role in tumor progression and metastasis; therefore, clarifying the regulation of EMT will greatly enhance our understanding of tumor migration and invasion. Accumulating evidence suggests that TAMs play a critical role in the regulation of EMT in cancers. TAM-derived factors play important roles in initiation and progression of the EMT [43].

Also of interest, based on results of studies on animal models, TAMs may play a role in forming premetastatic niches in organs to which the tumor will eventually metastasize. Specifically, TNF- $\alpha$, VEGF, and transforming growing factor- $\beta$ (TGF- $\beta$ ), which are derived from TAMs in cancer tissues, are believed to be transported through the bloodstream to destination organs, where they induce macrophages to produce S100A8 and serum amyloid A3. Both S100A8 and serum amyloid A3 can recruit macrophages and tumor cells to these organs and promote the formation of metastatic foci [44]. Thus, TAMs are believed to not only influence their local environments but also to influence macrophages throughout the body and thereby contribute to disease progression.

\section{Angiogenesis}

A few studies have shown that the levels of TAMs are closely associated with the number of vessels in human cancers. Hypoxia is a major driver of tumor angiogenesis. Accumulated macrophages can be found in hypoxic areas of tumor, and particularly in necrotic tissue. HIF$1 \alpha$, which is expressed in macrophages, regulates the transcription of many genes such as VEGF associated with angiogenesis at hypoxic sites. Genetic analysis has revealed that TAMs can produce VEGF, TNF- $\alpha$, IL-1 $\beta$, IL-8 (CXCL8), platelet-derived growth factor (PDGF), basic fibroblast growth factor (bFGF), thymidine phosphorylase, MMPs, and other molecules that are involved in tumor angiogenesis, indicating that TAMs promote the formation of intratumoral blood vessels that provide nutrition for tumor growth [45]. Tie $2^{+}$TAMs are closely associated with tumor vasculature and have been found crucial for angiogenesis in orthotopic and transgenic tumor models [46], which depend on endothelial cellproduced angiopoietin-2 (ANG2) and Tie2 receptors on TAMs along the vasculature (Fig. 2).

\section{Diagnostic biomarker of cancer}

As the relationship between TAMs and malignant tumors becomes clearer, TAMs have begun to be used from bench to bedside, including as potential biomarkers for diagnosis and prognosis of cancer and as therapeutic targets for cancer. First, we will explain how TAMs can be served as potential diagnostic biomarkers of cancer (Fig. 3). Human TAMs are commonly identified by expression of CD163, CD204, or CD206; these biomarkers are not specific for a particular type of cancer.

In our previous study, $\mathrm{CD} 163^{+} \mathrm{CD} 14^{+}$macrophages were determined to be potential immune diagnostic markers for malignant pleural effusion (MPE) and have better assay sensitivity than that of cytological analysis [47]. In addition, a serum CD163 value of $1.8 \mathrm{mg} / \mathrm{L}$ was set as a cutoff concentration in a survival analysis of patients with multiple myeloma and should be validated in future studies [48]. Tang reviewed the relationship between TAMs and clinicopathological parameters in human breast cancers and addressed the potential value of TAMs as diagnostic biomarkers [49].

Using precision microfilters under low-flow conditions, circulating cancer-associated macrophage-like cells were isolated from the peripheral blood of patients with breast, pancreatic, or prostate cancer. These cells, which are not found in healthy individuals, were found to express epithelial, monocytic, and endothelial protein markers and were observed bound to circulating tumor cells in circulation [50]. These data support the hypothesis that disseminated TAMs can be used as a biomarker of advanced disease, suggesting that TAMs play a participatory role in tumor cell migration. 


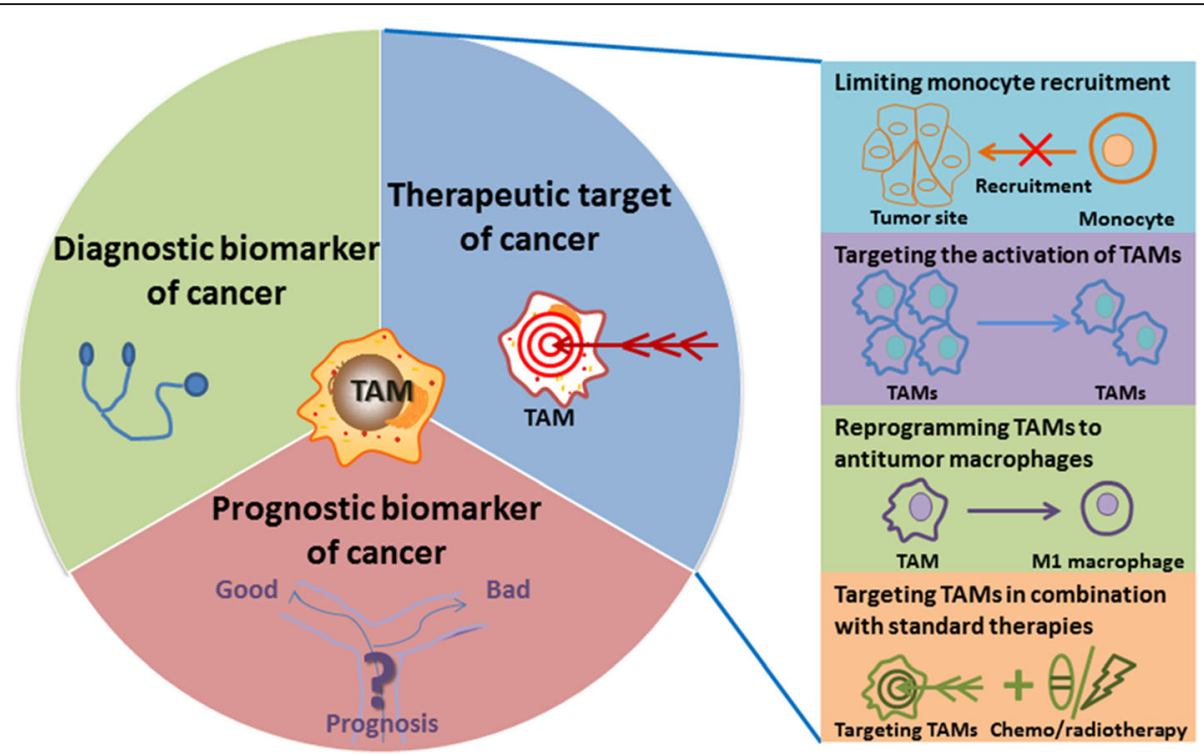

Fig. 3 The clinical application of TAMs. As the relationship between TAMs and malignant tumors becomes clearer, TAMs are beginning to be seen as potential biomarkers for diagnosis and prognosis of cancers and as therapeutic targets in cancers. Therapeutic strategies directed at TAMs can be grouped into four areas: limiting monocyte recruitment, targeting the activation of TAMs, reprogramming TAMs to antitumor macrophages, and targeting TAMs in combination with standard therapies

\section{Prognostic biomarker of cancer}

Due to TAMs' important role in tumor progression, the level of infiltrated TAMs may be used as a prognostic factor in cancers (Fig. 3). Over $80 \%$ of immunohistochemical studies using various human tumor tissues have shown that higher numbers of TAMs are associated with worse clinical prognosis. Recently, we showed that the accumulation of $\mathrm{CD}_{163^{+}}$TAMs in MPE caused by lung cancer was closely correlated with poor prognosis [51]. The results of a study indicate that CD204+ ${ }^{+}$TAMs are an independent prognostic factor in esophageal squamous cell carcinoma [52]. A high density of infiltrated TAMs is associated with aggressive features of gastric cancer and is an independent prognostic marker in gastric cancer patients [53]. Macrophage phenotypes (CD68, MAC387, and CLEVER-1/Stabilin-1) provide significant independent prognostic information, particularly in bladder cancers following transurethral resection [54]. Moreover, evidence suggests the expression of inflammation-related genes, especially genes related to polarization of TAMs, contributes to prognosis and is associated with poor clinical outcomes. Therefore, TAMs can be used as a potential biomarker for prognosis of cancers in clinics.

\section{Therapeutic targets in cancer}

As discussed above, there is strong evidence of tumor promotion by TAMs in different cancer models and an increased TAM prevalence correlates with low survival rates in many human cancers. Therefore, targeting TAMs is a novel strategy for the treatment of cancers.
Therapeutic strategies directed at TAMs can be grouped into four areas described as below (Fig. 3).

\section{Limiting monocyte recruitment}

One strategy for targeting TAMs is to block monocyte recruitment into tumor tissues. Targeting the CCL2CCR2 axis is promising due to its important role in monocyte recruitment in tumors. A CCL2-blocking agent (carlumab, CNTO88) has been shown to inhibit the growth of several cancers in animal models. A phase II study of carlumab in metastatic castration-resistant prostate cancer patients showed that this antibody was well tolerated, but that neither blocked the CCL2/CCR2 axis nor showed antitumor activity as a single agent in these metastatic cancer patients [55] (NCT00992186, Table 1). Similar results of Brana et al. showed that carlumab in combination with four chemotherapy regimens for the treatment of patients with solid tumors was well tolerated, although no long-term suppression of serum CCL2 or significant tumor responses were observed [56] (NCT01204996, Table 1). However, according to the results of other study, carlumab was well tolerated, with evidence of transient CCL2 suppression and preliminary antitumor activity [57] (NCT00537368, Table 1).

Sanford et al. demonstrate that a CCR2 antagonist (PF-04136309) can block the mobilization of CCR2 ${ }^{+}$ monocytes from bone marrow to tumors in a mouse model of pancreatic cancer and can lead to TAM depletion, causing the inhibition of tumor growth and distant metastasis [58]. PF-04136309, in combination with FOLFIRINOX 


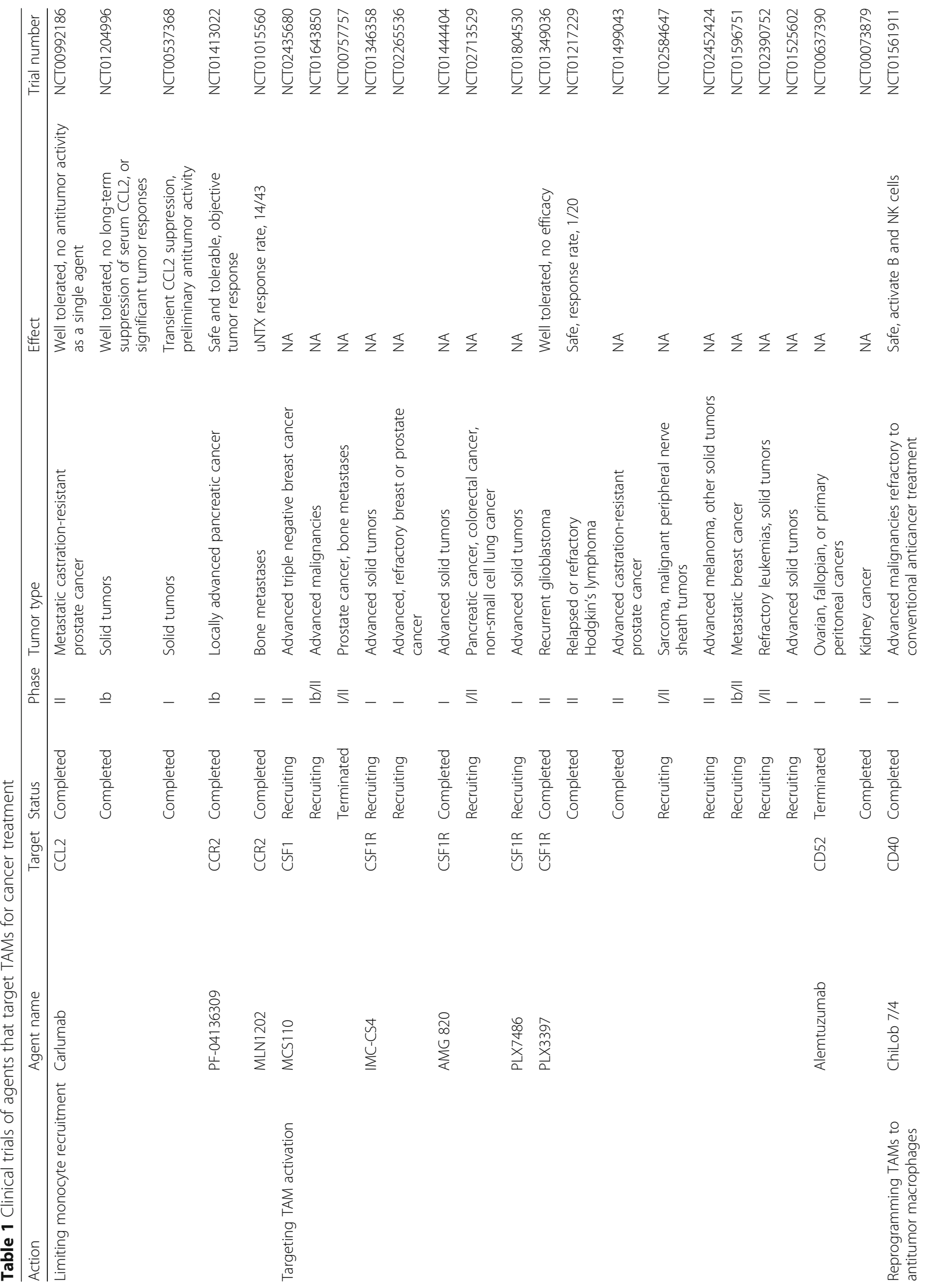




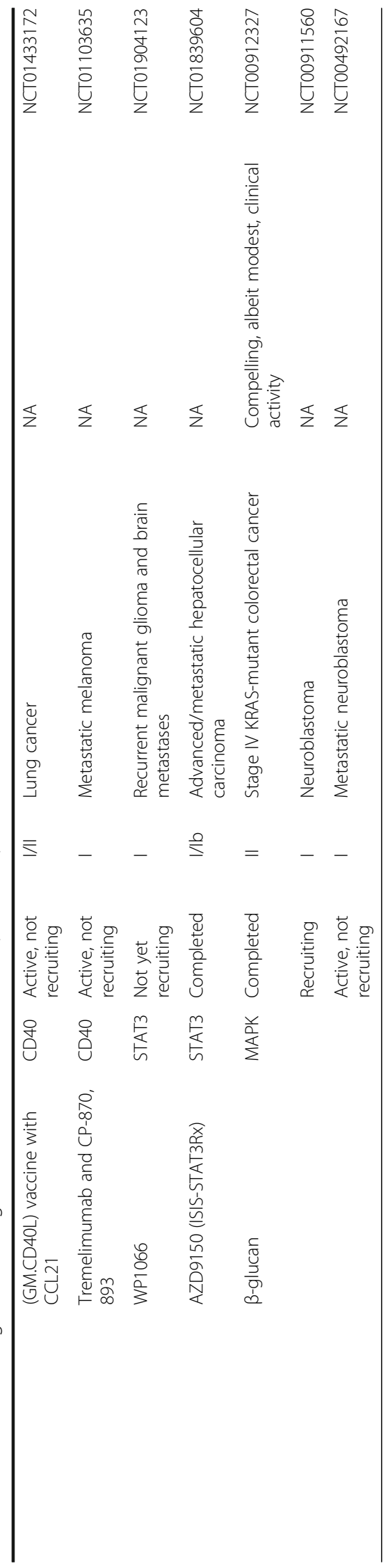


chemotherapy, was used in a phase Ib trial (NCT01413022, Table 1). This therapy was found safe and tolerable with an objective tumor response [59]. Moreover, the efficiency of the humanized antibody specific for CCR2 (MLN1202) was determined in a clinical investigation (NCT01015560, Table 1).

Treatment with systemic CD11b-neutralizing monoclonal antibodies has been shown to prevent the recruitment of myeloid cells to tumors. It has been shown that the use of Mac-1 (CD11b/CD18) antibodies leads to an improved response to radiation therapy in squamous cell carcinoma xenografts of mice, which is accompanied by reduced infiltration of myeloid cells expressing MMP-9 and S100A8 inside tumors [60].

Because targeting monocytes, prior to being recruited to tumors, has been effective in various cancer models and partial clinical trials, TAMs can be directly targeted as well by other approaches once they invade tumors.

\section{Targeting the activation of TAMs}

TAMs can be targeted at the level of activation using various strategies. CSF1/CSF1 receptor (CSF1R) signaling is critical for the generation of monocyte progenitors in bone marrow and TAM polarization in tumor tissues. For these reasons, CSF1/CSF1R signaling is an attractive target for cancer treatment. Genetic loss of CSF1 results in significantly reduced metastasis and delayed tumor progression in breast and neuroendocrine tumor models [61]. miR-26a expression reduces CSF1 expression in hepatocellular carcinoma [62]. Based on these results, several clinical trials of CSF1/CSF1R inhibitors have been completed or are ongoing (Table 1).

Macrophage surface markers can act as useful therapeutic targets. Mannose receptor CD206 can be exploited as a macrophage-specific target. A single-chain peptide bound to the CD206 receptor was attached to nanobodies that can selectively target CD206 ${ }^{+}$TAMs [63]. Legumain, a stress protein and a member of the asparagine endopeptidase family, can serve as an efficient therapeutic target when overexpressed in TAMs [64]. Targeting surface markers such as scavenger receptor A and CD52 by using immunotoxin-conjugated monoclonal antibodies (mAbs) has been investigated in ovarian cancer [65]. Moreover, the efficiency of alemtuzumab (anti-CD52 antibody) as a tumor treatment in ongoing clinical trials is under investigation (NCT00637390, NCT00073879, Table 1).

Trabectedin (ET743, Yondelis ${ }^{\circ}$ ) was shown to decrease the number of TAMs in tumor tissues by inducing apoptosis of monocytes and macrophages [66, 67]. Based on the favorable results of several phase I, II, and III clinical trials, trabectedin has gained full marketing approval from the European Commission for use in the treatment of ovarian cancer and soft tissue sarcomas and FDA approval in 2015 for use in unresectable or metastatic liposarcoma or leiomyosarcoma [68].

\section{Reprogramming TAMs to antitumor macrophages}

As discussed above, one of the key features of macrophages is their plasticity, which enables them to change their phenotype in the tumor microenvironment. Thus, reprogramming TAMs to an antitumor phenotype is an attractive therapeutic strategy. Antitumor macrophages are good at scavenging and destroying phagocytosed tumor cells [69]. The results of our previous study showed that pseudomonas aeruginosa mannose-sensitive hemagglutinin, which is used in MPE treatment, reeducated $\mathrm{CD}_{163^{+}}$TAMs to M1 macrophages in MPE, suggesting that reprogramming $\mathrm{CD}_{163^{+}}$TAMs can be served as a potential therapeutic strategy of MPE [51].

Nanoparticles are gradually used in polarization of TAMs into antitumor macrophages. Recently, Zanganeh et al. found that ferumoxytol significantly inhibited growth of subcutaneous adenocarcinomas in mice, and this tumor growth inhibition was accompanied by an increase in proinflammatory M1 macrophages in tumor tissues [70]. Recent data suggest that bioconjugated manganese dioxide nanoparticles enhance the responses of chemotherapy by inducing TAM toward M1-like phenotype [71]. Synthesized nanoparticles with IL-12 payload can reverse macrophages to antitumor function [72].

CD40 is a surface marker of macrophages that can be used to inhibit cytotoxic functions. The combination of a CD40 agonist with gemcitabine in unresectable pancreatic cancer resulted in regression of tumors by promoting antitumor macrophages [73]. ChiLob $7 / 4$ is an intermediate CD40 agonist and chimeric IgG1, which was also shown to induce pro-inflammatory cytokines, with promising results in CD40-expressing solid tumors and diffuse large B cell lymphoma resistant to conventional therapy in a phase I clinical trial [74] (NCT01561911, Table 1). Other clinical trials of molecules targeting CD40 for cancer treatment are ongoing (NCT01433172, NCT01103635, Table 1).

Activation of the NF- $\mathrm{kB}$ pathway also plays an important role in polarization of TAMs to an antitumor phenotype using TLR agonists, anti-CD40 mAbs, and IL-10 mAbs [75]. In addition, regulation of STAT1 activity is an attractive strategy to induce an antitumor phenotype in macrophages because of the increase production of IL-12 in a murine carcinoma model. A small molecule inhibitor of STAT3 (WP1066) was found to reverse immune tolerance in patients with malignant gliomas and to selectively induce the expression of costimulatory molecules CD80, CD86, and IL-12 on peripheral and tumor-infiltrating macrophages [76]. An investigation of this agent to treat recurrent malignant gliomas and brain metastasis is ongoing (NCT01904123, Table 1). 
Thymosin- $\alpha$ is an immunomodulating hormone that can reeducate TAMs into dendritic cells, which participate in antitumor host responses and produce high level of pro-inflammatory cytokines. Nanodelivery of thymosin- $\alpha$ is a feasible approach to increase immune activity in cancer patients. Moreover, several clinical trials have confirmed that thymosin- $\alpha$ prolongs survival in patients with metastatic melanomas and advanced non-small cell lung cancers [77].

$\beta$-glucan, a yeast-derived polysaccharide, has been shown to differentiate TAMs into an M1 phenotype and is a potent immunomodulator with anticancer properties [78]. The use of $\beta$-glucan is currently under investigation in a phase I clinical trial of patients with neuroblastoma [79] (NCT00911560, Table 1). In another clinical trial, a $\beta$-glucan polymer (PGG) showed compelling but modest activity in a phase II multi-cancer study [80] (NCT00912327, Table 1). Furthermore, the efficiency of $\beta$-glucan is currently under phase I clinical investigation (NCT00492167, Table 1).

\section{Targeting TAMs in combination with standard therapies}

Radiotherapy and chemotherapy are useful treatments in many cancers, and studies have shown that infiltrated myeloid increases after irradiation. However, the interaction between tumor cells and stroma after these therapies remains poorly defined. DNA damage, cell death, and increased hypoxia have been observed in tumors after radiotherapy, which has been shown to lead to macrophage recruitment and promote tumor progression in animal models [81]. Therefore, it is essential to combine TAM targeting with standard therapies for effective tumor treatment.

The HIF-1 pathway is stimulated by radiation-induced tumor hypoxia, and the HIF-1 inhibitor can result in decreased infiltration of myeloid cells into tumors [82]. Even more strikingly, blocking CSF1R signaling appears to enhance the efficacy of several other standard therapies. As such, CSF1R blockade has been shown to increase the efficacy of chemotherapy for pancreatic tumors [83].

\section{Conclusions}

In this review, we discussed the origin, polarization, function, and clinical application of TAMs. TAMs play critical roles in the development and progression of human cancers. Therefore, it will be critical to obtain a better understanding of TAMs to apply clinically, especially as a diagnosis and prognosis marker and a therapeutic target as well. Targeting TAMs is a promising strategy for cancer treatment. Recent ongoing experimental, preclinical, and clinical studies of TAMs have shown encouraging progress. We believe that TAM-targeted therapies will be applied in cancer patients in the future.

\section{Abbreviations}

ANG2: Angiopoietin-2; bFGF: Basic fibroblast growth factor; CCL: Chemokine (C-C motif) ligand; CCR: Chemokine (C-C motif) receptor; CSF: Colony-stimulating factor; CSF1R: CSF1 receptor; CTL: Cytotoxic T lymphocyte; CXCL: CXC motif chemokine ligand; CXCR: CXC chemokine receptor type; ECM: Extracellular matrix; EMT: Epithelial-mesenchymal transition; HIF: Hypoxia-inducible factor;

HMGB1: High-mobility group box 1 protein; Ig: Immunoglobulin; IL: Interleukin; iNOS: Inducible nitric oxide synthase; MDSCs: Myeloid-derived suppressor cells; MFG-E8: Milk fat globule-epithelial growth factor 8 protein; MIF: Migration inhibitory factor; MMP: Matrix metallopeptidase; MPE: Malignant pleural effusion; NF-kB: Nuclear factor kB; PDGF: Platelet-derived growth factor; PGE2: Prostaglandin E2; ROS: Reactive oxygen species; STAT: Signal transduction and transcription; TAMs: Tumor-associated macrophages; TGF- $\beta$ : Transforming growing factor- $\beta$; Th: Helper T cell; TLRs: Toll-like receptors; TNF-a: Tumor necrosis factor-a; Tregs: Regulatory T cells; VEGFA: Vascular endothelial growth factor A

\section{Acknowledgements}

Not applicable.

\section{Funding}

This study was supported by grants from the National Natural Science Foundation of China (No. 81171986, No. 81602024), Funding from State's Key Project of Research and Development Plan (No. 2016YFC1303500),

International Research Cooperation Grant from Science and Technology Department of Henan Province (No. 162102410059), Research Grant from the Ministry of Public Health (No. 201501004), Funds for Creative Research Team of Henan Province (No. C20120030), Creative Research Team of Higher Education of Henan Province.

\section{Availability of data and materials}

The material supporting the conclusion of this review has been included within the article.

\section{Authors' contributions}

LY wrote and edited the manuscript, YZ revised the manuscript. All authors read and approved final manuscript.

\section{Competing interests}

The authors declare that they have no competing interests.

\section{Consent for publication}

This is not applicable for this review.

Ethics approval and consent to participate

This is not applicable for this review.

\section{Author details}

${ }^{1}$ Biotherapy Center, The First Affiliated Hospital of Zhengzhou University, No.1 Jianshe East Road, Zhengzhou 450052, Henan Province, China. ${ }^{2}$ Cancer Center, The First Affiliated Hospital of Zhengzhou University, No.1 Jianshe East Road, Zhengzhou 450052, Henan Province, China. ${ }^{3}$ School of Life Science, Zhengzhou University, No.100 Kexue Road, Zhengzhou 450001, Henan Province, China.

Received: 23 January 2017 Accepted: 23 February 2017

Published online: 28 February 2017

\section{References}

1. Tu Z, Xiao R, Xiong J, Tembo KM, Deng X, Xiong M, Liu P, Wang M, Zhang Q. CCR9 in cancer: oncogenic role and therapeutic targeting. Hematol Oncol. 2016;9:10.

2. Caso G, Barry C, Patejunas G. Dysregulation of CXCL9 and reduced tumor growth in Egr-1 deficient mice. J Hematol Oncol. 2009;2:7.

3. Teng F, Tian WY, Wang YM, Zhang YF, Guo F, Zhao J, Gao C, Xue FX. Cancer-associated fibroblasts promote the progression of endometrial cancer via the SDF-1/CXCR4 axis. J Hematol Oncol. 2016;9:8.

4. Quail DF, Joyce JA. Microenvironmental regulation of tumor progression and metastasis. Nat Med. 2013;19:1423-37.

5. Chen Y, Zhang S, Wang Q, Zhang X. Tumor-recruited M2 macrophages promote gastric and breast cancer metastasis via M2 macrophage-secreted CHI3L1 protein. J Hematol Oncol. 2017;10:36. 
6. Grivennikov SI, Greten FR, Karin M. Immunity, inflammation, and cancer. Cell. 2010;140:883-99.

7. Van Overmeire E, Laoui D, Keirsse J, Bonelli S, Lahmar Q, Van Ginderachter JA. STAT of the union: dynamics of distinct tumor-associated macrophage subsets governed by STAT1. Eur J Immunol. 2014;44:2238-42.

8. De I, Steffen MD, Clark PA, Patros CJ, Sokn E, Bishop SM, Litscher S, Maklakova VI, Kuo JS, Rodriguez FJ, et al. CSF1 overexpression promotes high-grade glioma formation without impacting the polarization status of gliomaassociated microglia and macrophages. Cancer Res. 2016;76:2552-60.

9. Franklin RA, Liao W, Sarkar A, Kim MV, Bivona MR, Liu K, Pamer EG, Li MO. The cellular and molecular origin of tumor-associated macrophages. Science. 2014;344:921-5.

10. Linde N, Lederle W, Depner S, van Rooijen N, Gutschalk CM, Mueller MM. Vascular endothelial growth factor-induced skin carcinogenesis depends on recruitment and alternative activation of macrophages. J Pathol. 2012;227:17-28.

11. Su S, Liu Q, Chen J, Chen J, Chen F, He C, Huang D, Wu W, Lin L, Huang W, et al. A positive feedback loop between mesenchymal-like cancer cells and macrophages is essential to breast cancer metastasis. Cancer Cell. 2014;25:605-20.

12. Nandi B, Shapiro M, Samur MK, Pai C, Frank NY, Yoon C, Prabhala RH, Munshi NC, Gold JS. Stromal CCR6 drives tumor growth in a murine transplantable colon cancer through recruitment of tumor-promoting macrophages. Oncoimmunology. 2016;5:e1189052.

13. Mota JM, Leite CA, Souza LE, Melo PH, Nascimento DC, De-DeusWagatsuma VM, Temporal J, Figueiredo F, Noushmehr H, Alves-Filho JC, et al. Post-sepsis state induces tumor-associated macrophage accumulation through CXCR4/CXCL12 and favors tumor progression in mice. Cancer Immunol Res. 2016;4:312-22.

14. Tian X, Tian J, Tang X, Ma J, Wang S. Long non-coding RNAs in the regulation of myeloid cells. J Hematol Oncol. 2016;9:99.

15. Wang H, Shao Q, Sun J, Ma C, Gao W, Wang Q, Zhao L, Qu X. Interactions between colon cancer cells and tumor-infiltrated macrophages depending on cancer cell-derived colony stimulating factor 1. Oncoimmunology. 2016;5:e1122157.

16. Li Y, Zheng Y, Li T, Wang Q, Qian J, Lu Y, Zhang M, Bi E, Yang M, Reu F, et al. Chemokines CCL2, 3, 14 stimulate macrophage bone marrow homing, proliferation, and polarization in multiple myeloma. Oncotarget. 2015;6:24218-29.

17. Ambade A, Satishchandran A, Saha B, Gyongyosi B, Lowe P, Kodys K, Catalano D, Szabo G. Hepatocellular carcinoma is accelerated by NASH involving M2 macrophage polarization mediated by hif-1a induced IL-10. Oncoimmunology. 2016;5:e1221557.

18. Zhao P, Gao D, Wang Q, Song B, Shao Q, Sun J, Ji C, Li X, Li P, Qu X. Response gene to complement 32 (RGC-32) expression on M2-polarized and tumor-associated macrophages is M-CSF-dependent and enhanced by tumor-derived IL-4. Cell Mol Immunol. 2015;12:692-9.

19. Ma R, Ji T, Chen D, Dong W, Zhang H, Yin X, Ma J, Liang X, Zhang Y, Shen $G$, et al. Tumor cell-derived microparticles polarize M2 tumor-associated macrophages for tumor progression. Oncoimmunology. 2016;5:e1118599.

20. Cha HR, Lee JH, Hensel JA, Sawant AB, Davis BH, Lee CM, Deshane JS, Ponnazhagan S. Prostate cancer-derived cathelicidin-related antimicrobial peptide facilitates macrophage differentiation and polarization of immature myeloid progenitors to protumorigenic macrophages. Prostate. 2016;76:624-36.

21. Tripathi C, Tewari BN, Kanchan RK, Baghel KS, Nautiyal N, Shrivastava R, Kaur H, Bhatt ML, Bhadauria S. Macrophages are recruited to hypoxic tumor areas and acquire a pro-angiogenic M2-polarized phenotype via hypoxic cancer cell derived cytokines Oncostatin M and Eotaxin. Oncotarget. 2014;5:5350-68.

22. Xiao G, Wang X, Sheng J, Lu S, Yu X, Wu JD. Soluble NKG2D ligand promotes MDSC expansion and skews macrophage to the alternatively activated phenotype. J Hematol Oncol. 2015;8:13.

23. Gocheva V, Wang HW, Gadea BB, Shree T, Hunter KE, Garfall AL, Berman T, Joyce JA. IL-4 induces cathepsin protease activity in tumor-associated macrophages to promote cancer growth and invasion. Gene Dev. 2010;24:241-55.

24. DeNardo DG, Barreto JB, Andreu P, Vasquez L, Tawfik D, Kolhatkar N Coussens LM. CD4(+) T cells regulate pulmonary metastasis of mammary carcinomas by enhancing protumor properties of macrophages. Cancer Cell. 2009;16:91-102.
25. Andreu P, Johansson M, Affara NI, Pucci F, Tan T, Junankar S, Korets L, Lam J, Tawfik D, DeNardo DG, et al. FcRgamma activation regulates inflammationassociated squamous carcinogenesis. Cancer Cell. 2010;17:121-34.

26. Pedroza-Gonzalez A, Xu K, Wu TC, Aspord C, Tindle S, Marches F, Gallegos M, Burton EC, Savino D, Hori T, et al. Thymic stromal lymphopoietin fosters human breast tumor growth by promoting type 2 inflammation. Int J Clin Exp Med. 2011;208:479-90.

27. Yamada K, Uchiyama A, Uehara A, Perera B, Ogino S, Yokoyama Y, Takeuchi Y, Udey MC, Ishikawa O, Motegi S. MFG-E8 drives melanoma growth by stimulating mesenchymal stromal cell-induced angiogenesis and M2 polarization of tumor-associated macrophages. Cancer Res. 2016;76:4283-92.

28. Yaddanapudi K, Putty K, Rendon BE, Lamont GJ, Faughn JD, Satoskar A, Lasnik A, Eaton JW, Mitchell RA. Control of tumor-associated macrophage alternative activation by macrophage migration inhibitory factor. J Immunol. 2013:190:2984-93.

29. Sica A, Saccani A, Bottazzi B, Polentarutti N, Vecchi A, van Damme J, Mantovani A. Autocrine production of IL-10 mediates defective IL-12 production and NF-kappa B activation in tumor-associated macrophages. J Immunol. 2000;164:762-7.

30. Sánchez-Martín L, Estecha A, Samaniego R, Sánchez-Ramón S, Vega MÁ, Sánchez-Mateos $P$. The chemokine CXCL12 regulates monocytemacrophage differentiation and RUNX3 expression. Blood. 2011;117:88-97.

31. Doedens AL, Stockmann C, Rubinstein MP, Liao D, Zhang N, DeNardo DG, Coussens LM, Karin M, Goldrath AW, Johnson RS. Macrophage expression of hypoxia-inducible factor-1 alpha suppresses T-cell function and promotes tumor progression. Cancer Res. 2010;70:7465-75.

32. Kim S, Takahashi H, Lin WW, Descargues P, Grivennikov S, Kim Y, Luo JL, Karin M. Carcinoma-produced factors activate myeloid cells through TLR2 to stimulate metastasis. Nature. 2009;457:102-6.

33. Sorokin $L$. The impact of the extracellular matrix on inflammation. Nat Rev Immunol. 2010;10:712-23.

34. Raggi C, Mousa HS, Correnti M, Sica A, Invernizzi P. Cancer stem cells and tumor-associated macrophages: a roadmap for multitargeting strategies. Oncogene. 2016;35:671-82.

35. Grivennikov SI, Wang K, Mucida D, Stewart CA, Schnabl B, Jauch D, Taniguchi K, Yu GY, Osterreicher CH, Hung KE, et al. Adenoma-linked barrier defects and microbial products drive IL-23/L-17-mediated tumour growth. Nature. 2012:491:254-8.

36. Greten FR, Karin M. The IKK/NF-kappaB activation pathway - a target for prevention and treatment of cancer. Cancer Lett. 2004;206:193-9.

37. Kong L, Zhou Y, Bu H, Lv T, Shi Y, Yang J. Deletion of interleukin-6 in monocytes/macrophages suppresses the initiation of hepatocellular carcinoma in mice. J Exp Clin Canc Res. 2016;35:131.

38. Lu T, Ramakrishnan R, Altiok S, Youn Jl, Cheng P, Celis E, Pisarev V, Sherman S, Sporn MB, Gabrilovich D. Tumor-infiltrating myeloid cells induce tumor cell resistance to cytotoxic T cells in mice. J Clin Invest. 2011;121:4015-29.

39. Kuang DM, Zhao Q, Peng C, Xu J, Zhang JP, Wu C, Zheng L. Activated monocytes in peritumoral stroma of hepatocellular carcinoma foster immune privilege and disease progression through PD-L1. J Exp Med. 2009;206:1327-37.

40. Bingle L, Brown NJ, Lewis CE. The role of tumour-associated macrophages in tumour progression: implications for new anticancer therapies. J Pathol. 2002;196:254-65.

41. Chen J, Yao Y, Gong C, Yu F, Su S, Chen J, Liu B, Deng H, Wang F, Lin L, et al. CCL18 from tumor-associated macrophages promotes breast cancer metastasis via PITPNM3. Cancer Cell. 2011;19:541-55.

42. Finkernagel F, Reinartz $\mathrm{S}$, Lieber S, Adhikary T, Wortmann A, Hoffmann N, Bieringer T, Nist A, Stiewe T, Jansen JM, et al. The transcriptional signature of human ovarian carcinoma macrophages is associated with extracellular matrix reorganization. Oncotarget. 2016; doi: 10.18632/oncotarget.12180

43. Deng YR, Liu WB, Lian ZX, Li X, Hou X. Sorafenib inhibits macrophagemediated epithelial-mesenchymal transition in hepatocellular carcinoma. Oncotarget. 2016;7:38292-305.

44. Tomita T, Sakurai Y, Ishibashi S, Maru Y. Imbalance of Clara cell-mediated homeostatic inflammation is involved in lung metastasis. Oncogene. 2010; 30:3429-39.

45. Qian BZ, Pollard JW. Macrophage diversity enhances tumor progression and metastasis. Cell. 2010;141:39-51.

46. Mazzieri R, Pucci F, Moi D, Zonari E, Ranghetti A, Berti A, Politi LS, Gentner B, Brown JL, Naldini L, et al. Targeting the ANG2/TIE2 axis inhibits tumor growth and metastasis by impairing angiogenesis and disabling rebounds of proangiogenic myeloid cells. Cancer Cell. 2011;19:512-26. 
47. Wang F, Yang L, Gao Q, Huang L, Wang L, Wang J, Wang S, Zhang B, Zhang Y. CD163+CD14+ macrophages, a potential immune biomarker for malignant pleural effusion. Cancer Immunol Immunother. 2015;64:965-76.

48. Andersen MN, Abildgaard N, Maniecki MB, Møller HJ, Andersen NF. Monocyte/macrophage-derived soluble CD163: a novel biomarker in multiple myeloma. Eur J Haematol. 2014;93:41-7.

49. Tang X. Tumor-associated macrophages as potential diagnostic and prognostic biomarkers in breast cancer. Cancer Lett. 2013;332:3-10.

50. Adams DL, Martin SS, Alpaugh RK, Charpentier M, Tsai S, Bergan RC, Ogden IM, Catalona W, Chumsri S, Tang CM, et al. Circulating giant macrophages as a potential biomarker of solid tumors. Proc Natl Acad Sci U S A. 2014;111:3514-9.

51. Yang L, Wang F, Wang L, Huang L, Wang J, Zhang B, Zhang Y. CD163+ tumor-associated macrophage is a prognostic biomarker and is associated with therapeutic effect on malignant pleural effusion of lung cancer patients. Oncotarget. 2015:6:10592-603.

52. Shigeoka M, Urakawa N, Nakamura T, Nishio M, Watajima T, Kuroda D, Komori T, Kakeji Y, Semba S, Yokozaki H. Tumor associated macrophage expressing CD204 is associated with tumor aggressiveness of esophageal squamous cell carcinoma. Cancer Sci. 2013;104:1112-9.

53. Kim KJ, Wen XY, Yang HK, Kim WH, Kang GH. Prognostic implication of M2 macrophages are determined by the proportional balance of tumor associated macrophages and tumor infiltrating lymphocytes in microsatellite-unstable gastric carcinoma. PLoS One. 2015;10:e0144192.

54. Boström MM, Irjala H, Mirtti T, Taimen P, Kauko T, Ålgars A, Jalkanen S, Boström PJ. Tumor-associated macrophages provide significant prognostic information in urothelial bladder cancer. PLoS One. 2015;10:e0133552.

55. Pienta K, Machiels JP, Schrijvers D, Alekseev B, Shkolnik M, Crabb SJ, Li S, Seetharam S, Puchalski TA, Takimoto C, et al. Phase 2 study of carlumab (CNTO 888), a human monoclonal antibody against CC-chemokine ligand 2 (CCL2), in metastatic castration-resistant prostate cancer. Invest New Drugs. 2013;31:760-8.

56. Brana I, Calles A, LoRusso PM, Yee LK, Puchalski TA, Seetharam S, Zhong B, de Boer CJ, Tabernero J, Calvo E. Carlumab, an anti-C-C chemokine ligand 2 monoclonal antibody, in combination with four chemotherapy regimens for the treatment of patients with solid tumors: an open-label, multicenter phase 1b study. Target Oncol. 2015;10:111-23.

57. Sandhu SK, Papadopoulos K, Fong PC, Patnaik A, Messiou C, Olmos D, Wang G, Tromp BJ, Puchalski TA, Balkwill F, et al. A first-in-human, first-in-class, phase I study of carlumab (CNTO 888), a human monoclonal antibody against CC-chemokine ligand 2 in patients with solid tumors. Cancer Chemother Pharmacol. 2013;71:1041-50.

58. Germano G, Frapolli R, Belgiovine C, Anselmo A, Pesce S, Liguori M, Erba E, Uboldi S, Zucchetti M, Pasqualini F, et al. Role of macrophage targeting in the antitumor activity of trabectedin. Cancer Cell. 2013;23:249-62.

59. Sanford DE, Belt BA, Panni RZ, Mayer A, Deshpande AD, Carpenter D, Mitchem JB, Plambeck-Suess SM, Worley LA, Goetz BD, et al. Inflammatory monocyte mobilization decreases patient survival in pancreatic cancer: a role for targeting the CCL2/CCR2 axis. Clin Cancer Res. 2013;19:3404-15.

60. Nywening TM, Wang-Gillam A, Sanford DE, Belt BA, Panni RZ, Cusworth BM, Toriola AT, Nieman RK, Worley LA, Yano M, et al. Targeting tumourassociated macrophages with CCR2 inhibition in combination with FOLFIRINOX in patients with borderline resectable and locally advanced pancreatic cancer: a single-centre, open-label, dose-finding, nonrandomised, phase 1b trial. Lancet Oncol. 2016;17:651-62.

61. Ahn GO, Tseng D, Liao CH, Dorie MJ, Czechowicz A, Brown JM. Inhibition of Mac-1 (CD11b/CD18) enhances tumor response to radiation by reducing myeloid cell recruitment. Proc Natl Acad Sci U S A. 2010;107:8363-8.

62. Chai ZT, Zhu XD, Ao JY, Wang WQ, Gao DM, Kong J, Zhang N, Zhang YY, Ye $B G, M a D N$, et al. microRNA-26a suppresses recruitment of macrophages by down-regulating macrophage colony-stimulating factor expression through the PI3K/Akt pathway in hepatocellular carcinoma. J Hematol Oncol. 2015;8:56.

63. Pyonteck SM, Gadea BB, Wang HW, Gocheva V, Hunter KE, Tang LH, Joyce JA. Deficiency of the macrophage growth factor CSF-1 disrupts pancreatic neuroendocrine tumor development. Oncogene. 2012;31:1459-67.

64. Movahedi K, Schoonooghe S, Laoui D, Houbracken I, Waelput W, Breckpot K, Bouwens L, Lahoutte T, De Baetselier P, Raes G, et al. Nanobody-based targeting of the macrophage mannose receptor for effective in vivo imaging of tumor-associated macrophages. Cancer Res. 2012:72:4165-77.

65. Smahel M, Duskova M, Polakova I, Musil J. Enhancement of DNA vaccine potency against legumain. J Immunother. 2014:37:293-303.
66. Pulaski HL, Spahlinger G, Silva IA, McLean K, Kueck AS, Reynolds RK, Coukos G, Conejo-Garcia JR, Buckanovich RJ. Identifying alemtuzumab as an antimyeloid cell antiangiogenic therapy for the treatment of ovarian cancer. J Transl Med. 2009;7:49.

67. Allavena P, Signorelli M, Chieppa M, Erba E, Bianchi G, Marchesi F, Olimpio CO, Bonardi C, Garbi A, Lissoni A, et al. Anti-inflammatory properties of the novel antitumor agent yondelis (trabectedin): inhibition of macrophage differentiation and cytokine production. Cancer Res. 2005;65:2964-71.

68. Gordon EM, Sankhala KK, Chawla N, Chawla SP. Trabectedin for soft tissue sarcoma: current status and future perspectives. Adv Ther. 2016;33:1055-71.

69. Liu X, Kwon H, Li Z, Fu YX. Is CD47 an innate immune checkpoint for tumor evasion? J Hematol Oncol. 2017;10:12.

70. Zanganeh S, Hutter G, Spitler R, Lenkov O, Mahmoudi M, Shaw A, Pajarinen JS, Nejadnik H, Goodman S, Moseley M, et al. Iron oxide nanoparticles inhibit tumour growth by inducing pro-inflammatory macrophage polarization in tumour tissues. Nat Nanotechnol. 2016;11:986-94.

71. Song M, Liu T, Shi C, Zhang X, Chen X. Bioconjugated manganese dioxide nanoparticles enhance chemotherapy response by priming tumorassociated macrophages toward M1-like phenotype and attenuating tumor hypoxia. ACS Nano. 2016;10:633-47.

72. Wang Y, Lin YX, Qiao SL, An HW, Ma Y, Qiao ZY, Rajapaksha RP, Wang H. Polymeric nanoparticles promote macrophage reversal from $M 2$ to $M 1$ phenotypes in the tumor microenvironment. Biomaterials. 2017;112:153-63.

73. Beatty GL, Chiorean EG, Fishman MP, Saboury B, Teitelbaum UR, Sun W, Huhn RD, Song W, Li D, Sharp LL, et al. CD40 agonists alter tumor stroma and show efficacy against pancreatic carcinoma in mice and humans. Science. 2011;331:1612-6.

74. Johnson P, Challis R, Chowdhury F, Gao Y, Harvey M, Geldart T, Kerr P, Chan C, Smith A, Steven N, et al. Clinical and biological effects of an agonist anti-CD40 antibody: a Cancer Research UK phase I study. Clin Cancer Res. 2015;21:1321-8.

75. Seya T, Shime H, Matsumoto M. TAMable tumor-associated macrophages in response to innate RNA sensing. Oncoimmunology. 2012;1:1000-1.

76. Hussain SF, Kong LY, Jordan J, Conrad C, Madden T, Fokt I, Priebe W, Heimberger AB. A novel small molecule inhibitor of signal transducers and activators of transcription 3 reverses immune tolerance in malignant glioma patients. Cancer Res. 2007;67:9630-6.

77. Garaci E, Pica F, Serafino A, Balestrieri E, Matteucci C, Moroni G, Sorrentino R, Zonfrillo M, Pierimarchi P, Sinibaldi-Vallebona P. Thymosin alpha1 and cancer: action on immune effector and tumor target cells. Ann N Y Acad Sci. 2012;1269:26-33.

78. Chan GC, Chan WK, Sze DM. The effects of beta-glucan on human immune and cancer cells. J Hematol Oncol. 2009;2:25.

79. Kushner BH, Cheung IY, Modak S, Kramer K, Ragupathi G, Cheung NK. Phase I trial of a bivalent gangliosides vaccine in combination with beta-glucan for high-risk neuroblastoma in second or later remission. Clin Cancer Res. 2014;20:1375-82.

80. Segal NH, Gada P, Senzer N, Gargano MA, Patchen ML, Saltz LB. A phase II efficacy and safety, open-label, multicenter study of imprime PGG injection in combination with cetuximab in patients with stage IV KRAS-mutant colorectal cancer. Clin Colorectal Canc. 2016;15:222-7.

81. De Palma M, Lewis CE. Macrophage regulation of tumor responses to anticancer therapies. Cancer Cell. 2013;23:277-86.

82. Kioi M, Vogel H, Schultz G, Hoffman RM, Harsh GR, Brown JM. Inhibition of vasculogenesis, but not angiogenesis, prevents the recurrence of glioblastoma after irradiation in mice. J Clin Invest. 2010;120:694-705.

83. Mitchem JB, Brennan DJ, Knolhoff BL, Belt BA, Zhu Y, Sanford DE, Belaygorod L, Carpenter D, Collins L, Piwnica-Worms D, et al. Targeting tumor-infiltrating macrophages decreases tumor-initiating cells, relieves immunosuppression, and improves chemotherapeutic responses. Cancer Res. 2013;73:1128-41. 